\title{
Building Wireless Sensor Network Applications with LooCl
}

\author{
Daniel Hughes* \\ Computer Science and Software Engineering, Xi'an Jiaotong-Liverpool University, \\ Suzhou Industrial Park, Suzhou, Jiangsu Province, China, 215123 \\ daniel.hughes@xjtlu.edu.cn
}

Klaas Thoelen, Wouter Horré, Nelson Matthys, Javier Del Cid, Sam Michiels, Christophe Huygens, Wouter Joosen

IBBT-Distrinet, Department of Computer Science, Katholieke Universiteit Leuven

Celestijnenlaan 200A, B-3001, Heverlee, Leuven, Belgium.

\{firstname.lastname\}@cs.kuleuven.be

\section{Jo Ueyama}

Institute of Mathematics and Computer Science (ICMC), University of São Paulo, São Carlos, Brazil, 13566-585.

joueyama@usp.br

\begin{abstract}
Considerable research has been performed in applying run-time reconfigurable component models to the domain of wireless sensor networks. The ability to dynamically deploy and reconfigure software components has clear advantages in sensor networks, which are typically large in scale and expected to operate for long periods in the face of node mobility, dynamic environmental conditions and changing application requirements. LooCI is a component and binding model that is optimized for use in resource-constrained environments such as Wireless Sensor Networks. LooCI components use a novel event-based binding model that allows developers to model rich component interactions while providing support for run-time reconfiguration, reflection and policy-based management. This paper reports on the design of LooCI and describes a prototype implementation for the Sun SPOT. This platform is then evaluated in the context of a real-world river monitoring and warning scenario in the city of São Carlos, Brazil.
\end{abstract}

\section{KEYWORDS}

Wireless Sensor Networks, Component Based Software Engineering, Policy-based Management, Environmental Monitoring.

\section{INTRODUCTION}

Wireless Sensor Networks (WSNs), composed of embedded computers equipped with low power radios and low-cost sensors are being employed to support a growing range of fixed and mobile applications such as habitat monitoring [1], flood warning [2], industrial process control [3], disaster management [4] and in particular environmental monitoring [36]. WSNs are typically large in scale, subject to unreliable networking, node mobility, high risk of node failure 
and are expected to operate unattended for long periods. Recently, lightweight component models [4] [5] [6] [7] have emerged as a promising approach to managing complexity in WSN environments.

This paper describes the Loosely-coupled Component Infrastructure (LooCI), which features a loosely-coupled, event-based binding model inspired by event-driven programming models [6], Service Oriented Architectures (SOA) [8], publish-subscribe interaction models [9] and pluggable networking support [10]. The resulting architecture is light-weight and promotes a loose coupling between software components while facilitating advanced features such as policybased management, generic fault tolerance and distributed garbage collection.

We have evaluated LooCI in a real-world river monitoring case-study in the city of São Carlos in São Paulo state, Brazil. In this scenario, four WSN motes were deployed to monitor river health and build a better understanding of the relationship between two related creeks (one is a tributary of the other). This case-study allowed for a detailed evaluation of our WSN platform and provided valuable data to our local environmental science partners. In this study four environmental factors were monitored over a two-week period:

- Water depth is monitored using a hydrostatic level sensor in order to provide early warning of flood events.

- Water conductivity levels are monitored using a standard conductivity sensor in order to infer pollution levels.

- Methane levels are monitored using a simple $\mathrm{CH} 4$ sensor in order to detect decaying organic matter.

- Tamper detection is implemented using a three dimensional accelerometer.

The remainder of this paper is structured as follows. Section 2 discusses related work in the field of middleware for WSNs. Section 3 presents the LooCI middleware. Section 4 provides a static evaluation of the footprint and performance of the LooCI middleware. Section 5 evaluates the benefits of LooCI in a river monitoring case-study. Section 6 discusses directions for future work. Finally, Section 7 concludes.

\section{RELATED WORK}

A number of lightweight component models have been proposed for networked embedded scenarios. Each of these component models are discussed in Section 2.1. Related work on interaction models for WSN is then discussed in Section 2.3. Finally, Section 2.3 concludes by discussing other significant and related middleware approaches for WSN.

\subsection{Component Models for Wireless Sensor Networks}

NesC [6] is perhaps the best known and most widely deployed component model for WSN and is used to implement the TinyOS operating system [12]. NesC provides an eventdriven programming approach together with a static component model. The static nature of the component model means that unlike OpenCOM [7], RUNES [4], OSGi [5] or K-Components [30], NesC components cannot be dynamically rewired to support reconfiguration. Thus modification of a NesC composition after deploy-time necessitates complete replacement of the code image on each mote and re-starting of the application. The NesC binding model is based upon statically declared bidirectional component interfaces. This static programming approach allows for whole-program analysis and optimization [6], which is advantageous in resource constrained WSN environments. In terms of remote communication, TinyOS provides an 
implementation of the Active Messages paradigm [13]. Active Messages integrate communication and computation by incorporating a reference to a user-handler in each message. This allows for an event-based handler invocation model and prevents processes blocking while waiting for incoming messages. While TinyOS itself provides no support for remote bindings, extensions have been proposed to support traditional RPC-type bindings [14].

OpenCOM [7] is a general purpose, run-time reconfigurable component model. While it does not target WSN applications specifically, it has been deployed in a number of WSN scenarios [2] [15] and was used to implement the GridStix [15] WSN platform. OpenCOM features a small, platform independent run-time kernel that supports both static and dynamic compositions. In the case of static compositions, the kernel performs component linking at runtime and then exits, reducing overhead. In dynamic systems, the kernel persists and may be used to support rich run-time reconfiguration. OpenCOM also provides Component Frameworks (CFs) [7], which are used to provide support for the modeling the interactions between cooperating components. CFs may be local or distributed and can be used as a tool to reason about dynamic reconfiguration. OpenCOM component interfaces are specified using OMG Interface Definition Language (IDL). OpenCOM offers a flexible binding model, in which bindings themselves are modeled as components, allowing for advanced operations such as interception and reflection. While OpenCOM notionally supports diverse binding types, all current implementations use RPC-type bindings. Furthermore, while the OpenCOM component model is rich, the OpenCOM kernel alone consumes 32KB of RAM [7] and the entire GridStix WSN profile consumes more than 104KB [10]. This is significantly more memory than is available on many embedded sensor motes such as the popular T-Mote [16] or Mica-Z [17] platforms.

Reconfigurable Ubiquitous Networked Embedded Systems (RUNES) [4] brings OpenCOM functionality to more embedded devices. As with OpenCOM, RUNES has been realized in both $\mathrm{C}$ and Java. However, unlike OpenCOM, RUNES interface definitions are platform-specific. In the case of the $\mathrm{C}$ implementation structs are used, while in the case of the Java implementation standard Java interfaces are used. In addition to offering a smaller footprint than OpenCOM 2.0, RUNES also adds a number of inspection-based API calls to the OpenCOM kernel. As with OpenCOM, RUNES allows for the use of different binding types, however, all current implementations use RPC calls [4]. The Contiki [18] implementation of the Kernel consumes less than $1 \mathrm{~KB}$ of RAM, while the Unix/C and Java versions consume less than $20 \mathrm{~KB}$ [4]. The significantly smaller size of the Contiki version is achieved through extensive re-use of native Contiki concepts and therefore a direct size comparison is somewhat artificial.

The OSGi [5] specification defines a standardized, component based, computing environment for networked embedded devices that is used as the basis for an enhanced Service Oriented Architecture (SOA). OSGi also provides a secure execution environment, support for run-time reconfiguration, lifecycle management and various system services. OSGi is targeted at powerful embedded devices such as smart phones and network gateways along with desktop and enterprise computers. OSGi interfaces are modeled using SCA [23] and, as with OpenCOM [7] and RUNES [4], OSGi offers RPC-based bindings. Unfortunately, while OSGi is suitable for powerful embedded devices, the smallest implementation, Concierge [5] consumes more than $80 \mathrm{~KB}$, making it unsuitable for very resource constrained devices such as the T-Mote [16]. Unlike OpenCOM, OSGi offers no inherent support for distributed reconfiguration. 
R-OSGi [31] is a distributed middleware platform that extends the standard OSGi middleware by incorporating support for distributed reconfiguration. R-OSGi allows these distributed OSGi compositions to be managed in the same way as standard OSGi components.

The K-Component model [30] is a framework for building self-adaptive systems that operate in a decentralized environment. This model provides an Interface Definition Language (K-IDL), which is an extension to OMG IDL that supports the definition of component states and adaptation. K-Components provide lightweight support for dynamic software architectures and run-time reconfiguration. In K-Components, adaptation contracts define how components may be reconfigured. This is similar to the role fulfilled by CFs and Meta Object Protocols in OpenCOM [7] or R-OSGi [31] for OSGi [5]. Like OpenCOM, K-Components explicitly model connections as components which can be directly manipulated in order to bind and unbind components [19]. Unlike the previously discussed component models, the K-Component model only allows for local bindings. As K-Components provide no specific support for distributed binding or reconfiguration, this model is unsuitable for the creation of WSN applications that require distributed coordination.

\subsection{Interaction Models for Wireless Sensor Networks}

In [19] Parlavantzas et al. present a component-based model that can be used to define diverse forms of component bindings including: RPC, publish/subscribe, shared data spaces and pipes. In this model, binding types are expressed as UML collaborations which are modeled using 11 standard components. While this model is very flexible, this comes at a significant cost in terms of complexity and, as will be argued in Section 3.4, only a subset of binding are a good fit with WSN environments.

In [14] May et al. present an RPC extension to NesC [6] which allows for remote procedure calls (RPC) with similar semantics to local NesC calls. These RPC calls also support a simple one-hop service discovery scheme. This scheme allows developers to call methods on a neighbor, rather than targeting calls to a specific mote address. This reduces the burden on developers, who need not deal solely in terms of individual motes, and allows for simple fault tolerance in the presence of node failure or high levels of mobility. When an RPC call is made to a node's neighbors, the call will be served by exactly one neighbor, in an anycast fashion. This approach however has a number of significant limitations. Firstly, the lack of a common event or service description model complicates discovery of third party services and monitoring of interactions. Secondly, this approach still requires that developers model component interactions primarily on a mote-by-mote basis, which we believe is inefficient, if not infeasible, in largescale or mobile WSNs.

Pohl et al. [32] apply loosely-coupled service bindings to the problem of industrial automation and control on a powerful sensor and actuator platform. This paper argues that loosely coupled services are a promising model for binding components on sensor networks, as they promote interoperability, service discovery and allow for the re-wiring of bindings at run time. However, the Web Service approach used by DPWS is too heavy-weight to be feasible in more embedded WSN environments.

Jini [26] provides an event based Service Oriented Architecture for Java which leverages on RMI. Jini improves on the RMI registry by making the lookup service distributed and allowing clients to search for services based on their type, name or description. While Jini promotes service discovery and re-use, the underlying network implementation is based on RMI and TCP/IP, which are a poor fit with unreliable WSN network environments. Furthermore, the 
smallest Jini implementation [26] has a footprint of over 1MB, making it unsuitable for highly resource constrained devices.

\subsection{Other Middleware Approaches for Wireless Sensor Networks}

TeenyLIME [27] provides a tuple space abstraction in which processes communicate by writing and reading tuples into a shared virtual memory space. To support mobile applications, the tuple space in TeenyLIME is only shared by one-hop neighbours, though multi-hop communication is possible by traversing multiple tuple-spaces, this limits the scope of possible interactions between software components. Furthermore, in contrast with event based approaches, tuple spaces require active polling to receive data updates. This typically leads to higher communication overhead than event based approaches.

Impala [32] provides a suite of tools for installing and storing code updates. This includes a code distribution service and an 'application adapter' that adapts the behavior of a running application to the current state of the mote and to changes in the environment. This may include: the number of direct neighbors, available battery power and so on. Unfortunately, Impala offers limited support for the introduction of new functionality into the system at run-time, limiting the scope of reconfiguration.

TinyCubus [33] consists of a data management framework, a configuration engine and a cross-layer framework. A data management framework adapts both system and application elements according to certain criteria. A configuration engine provides the technology and the code distribution service needed to support reprogramming of the nodes. The code distribution service uses application knowledge about the sensor topology to optimize its behavior. The sharing of this knowledge is facilitated through the use of a cross-layer framework. Unfortunately, TinyCubus does not allow for the introduction of new functionality at run-time.

\section{THE LOOCI MIDDLEWARE}

The LooCI middleware is designed for Java devices such as the Sun SPOT [11] and Sentilla Perk [21]. As these platforms support standard Java ME [22], they reduce the burden on developers compared to bespoke WSN technologies such as TinyOS [12] or Contiki [18]. In a broader sense, the new generation of Java sensor motes opens the field of WSN development to millions of existing Java ME developers [22]. LooCI aims to bring the many advantages of reconfigurable, component-based software to Java-based WSN platforms, while preserving the benefits of familiarity and ease-of-use that Java ME offers. LooCI accomplishes this through the introduction of an easy-to-use component model, a simple yet extensible networking framework and a common event bus abstraction that provides a mechanism to connect software components for any kind of data exchange.

These features are discussed in sections 3.1 to 3.4 respectively. Section 3.5 then discusses PMA [35], a policy-based management architecture that has been developed for LooCI. An overview of the LooCI middleware for the Sun SPOT platform is shown in Figure 1.

\subsection{The LooCl Component Model}

The LooCI Component Model offers support for two component types, macrocomponents and microcomponents:

Macrocomponents are coarse-grained and service-like, building upon the notion of Isolates [25] inherent in the Sentilla [21] and SQUAWK [11] virtual machines. Isolates are process-like units of encapsulation that provide varying levels of control over their execution 
(exactly what is provided is dependant on the specific JVM). LooCI standardizes and extends the functionality offered by Isolates. Each macrocomponent runs in a separate isolate and communicates with the run-time middleware and other components via Inter Isolate RPC (IIRPC), which is offered by the underlying SQUAWK VM. Unlike microcomponents, macrocomponents may be multi-threaded and may include utility libraries and data files.

Microcomponents are fine-grained and self contained. All microcomponents run in the master Isolate alongside the LooCI runtime. Unlike macrocomponents, microcomponents must be single threaded and self contained, using no utility libraries or files. Aside from these restrictions, microcomponents offer identical functionality to macrocomponents with lower memory consumption and better performance.

Both macrocomponents and microcomponents offer run-time reconfiguration, interface definitions, introspection and support for the re-wiring of bindings. Each LooCI component has a unique identifier which is generated based upon the interfaces and dependencies of the component.

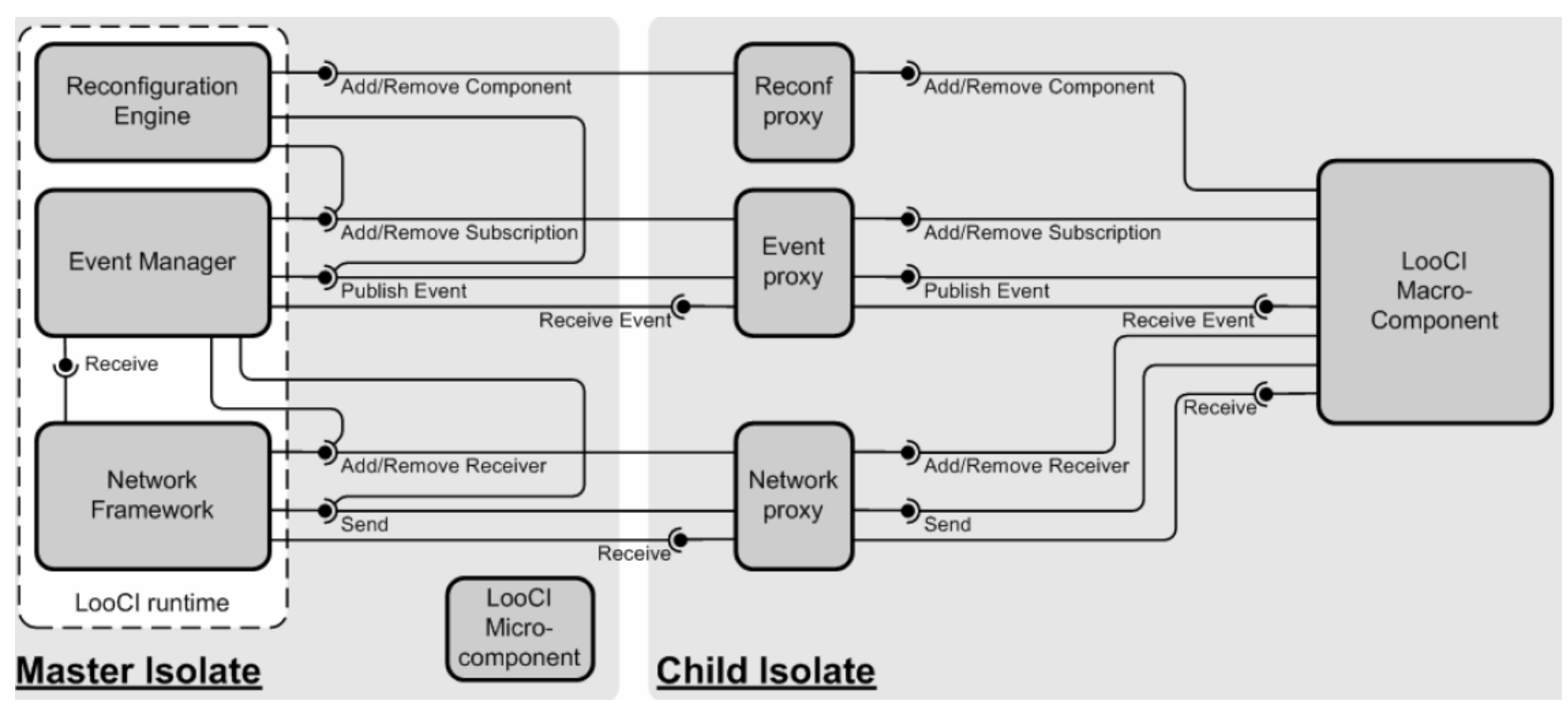

Figure 1: The Loosely Coupled Component Infrastructure (LooCI). LooCI Microcomponents provide and use the same interfaces as Macrocomponents but are directly connected to the LooCI runtime.

\subsubsection{Component Implementation}

In order to implement a LooCI component, developers extend a generic component baseclass, which provides standard methods to START and STOP a component as well as methods to place the component into quiescent state (MAKE_QUIESCENT) and RESUME operation from quiescent mode. For simple component implementations the control methods provided by the base-class are sufficient, however, for components with more complex requirements, developers may override these methods with their own implementations of START, STOP, MAKE_QUIESCENT and RESUME. Extending the component base-class also provides implicit access to the LooCI Network Framework and Event Bus. LooCI components may be deployed on demand via a LooCI application known as the Network Manager (see Figure 2), a 
maintenance application that has responsibility for every node in its associated WSN and provides facilities to internetwork with remote WSNs.

Implementing LooCI component has a very low overhead. In the simplest case, converting a standard Java ME CLDC 1.1 application into a LooCI component requires only that a single 'extends' statement be modified and that one method call - initialize() be added to the constructor.

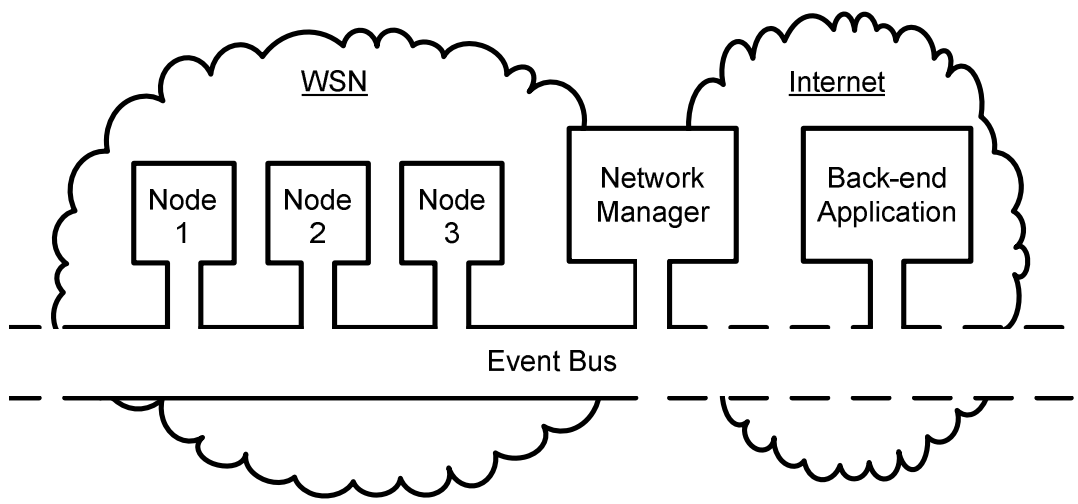

Figure 2: WSN and Back-End Integration

\subsubsection{Run-time Reconfiguration and Introspection}

Upon deployment, all LooCI components register with the per-node Reconfiguration Engine. The Reconfiguration Engine maintains a reference to all LooCI components running on the node and exposes a remote reconfiguration interface on the event bus. This allows run-time reconfiguration to be easily enacted by LooCI components that may be running on sensor motes, gateways or back-end devices.

The Reconfiguration Engine provides introspection at the node, component and binding level. At the node level, it is possible to inspect the LooCI components that are deployed on a mote. At the component level, the state of components can be inspected (states include STARTED, STOPPED, QUIESCENT etc.). At the binding level, the addresses to which a component is bound can be inspected. As with run-time reconfiguration, introspective information is accessed via the Reconfiguration Engine.

\subsubsection{Interface Definitions and Wiring}

LooCI components define their provided interfaces as the set of LooCI events that they publish. The receptacles of a LooCI component are similarly defined as the events to which they subscribe. Each LooCI event has a globally unique identifier (GUID) which classifies the event in terms of a global descriptive hierarchy (as described in Section 3.3).

Wiring of components occurs after deployment via the per-node Reconfiguration Engine, which exposes WIRE and UNWIRE operations. These mechanisms take as their argument a LooCI Component ID, an Event ID and a network address and remote Component ID to which the specified interface should be bound or unbound. LooCI addresses may map to a single node, a group of nodes, or an entire WSN. Wiring can be inspected at any time through the introspection API and re-wiring may be enacted by any element on the event bus.

As LooCI components communicate indirectly over the event bus, it is easy to build complex and flexible relationships between individual nodes, groups of nodes, or entire networks. Furthermore, re-wiring operations are low cost and easily enacted. As stated 
previously, this loose coupling is well suited to mobile environments. Furthermore, in conjunction with a global event classification, the event bus allows for advanced features like generic fault tolerance and distributed garbage collection as will be explored in section 3.4.4.

\subsection{Supporting Network Framework}

The LooCI Network Framework abstracts over and extends the networking services provided by the various underlying sensor platforms [11] [21] and provides a simple, uniform API to the upper middleware layers. This abstraction is supported by an extensible set of networking components, as shown in Figure 3.

The initial set of networking components consists of a UnicastComponent (UC) which provides both reliable and unreliable point-to-point multi-hop communication, a BroadcastComponent (BC) which implements network-wide broadcast and a NeighbourcastComponent (NC) which implements one-hop broadcast. The interface to the upper layers is agnostic to the various underlying communication paradigms and provides a simple send(message,destination) interface. Based upon the address provided and flags in the message header, the Network Framework automatically selects the most appropriate networking component to dispatch the message, as shown in Figure 3.

Incoming messages are passed from the underlying JVM to the Receiver component which in the case of reliable unicast responds with an ACK. As with Active Messages [13], LooCI messages are dispatched to the upper layers using an event-based handler invocation model. This approach avoids the need for connection setup and encourages programmers to think in an event-based fashion that we believe is appropriate for WSN (we explore this issue further in section 3.4). In the future, we intend to provide a more detailed description of the LooCI Network Framework, however, we consider such a description outside of the scope of this paper.

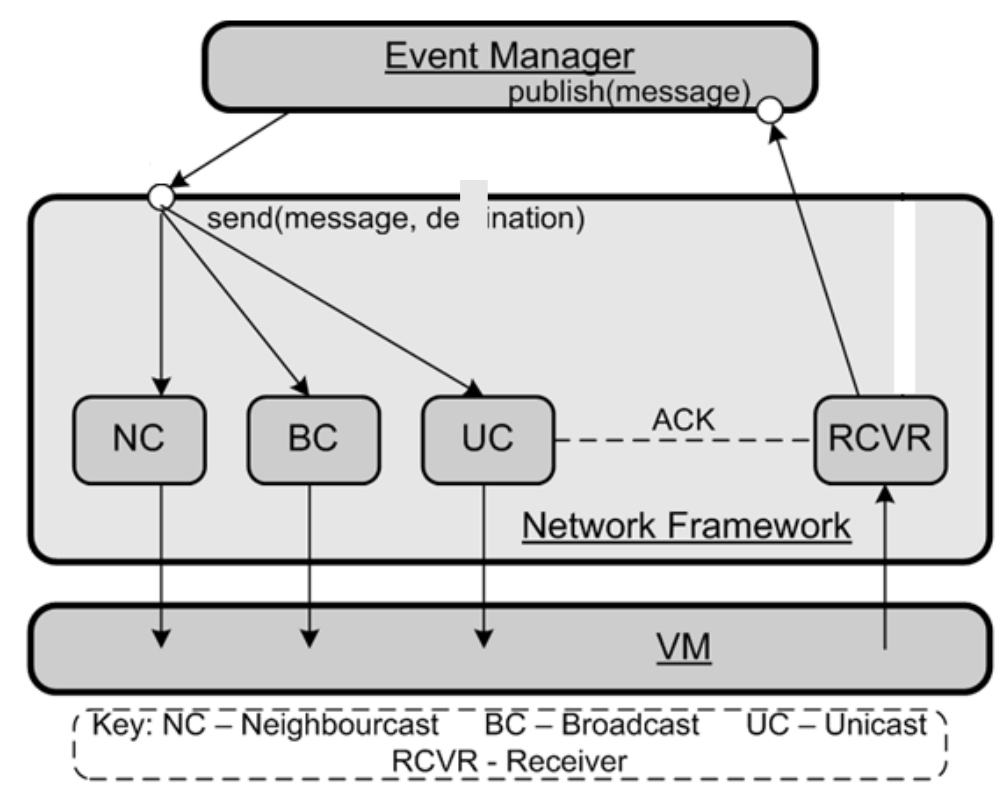

Figure 3: Supporting Network Framework

\subsection{The LooCl Event Manger}

The LooCI Event Manager embodies a generic communication substrate for disseminating events of any kind. This includes, but is not limited to: sensor readings, 
reconfiguration messages and state information. In concert with the networking framework described in the previous section, the Event Manager forms a distributed 'Event Bus' to which all LooCI components are connected. A per-node instance of the LooCI Event Manager implements a simple topic-based publish-subscribe event model, wherein events are disseminated to subscribers based upon their type. For example, a software element may subscribe to events of type 'TEMPERATURE' and may then be wired to a component at a given network location (local, a remote node or a remote group of nodes) that produces the required events. The interface to the event bus is simple and lightweight, however, in concert with LooCI's global, hierarchical type system, the Event Manager allows for rich modeling of interactions between nodes.

When an event is disseminated from a local interface, the publish(message) method of the EventManager will be called, causing the event to first be disseminated to all local components that have registered an interest in that event type. The event will then be disseminated to all remote components to which the associated interface is bound using the network framework described in Section 3.2.

When an event destined for the local node is received via the NetworkFramework, it will be disseminated to all local components that have registered an interest in that event type again using the publish(message) method of the EventManager.

Available components and interfaces may be discovered directly, by interrogating the introspection interface of individual motes or, more commonly, through the Network Manager of a WSN (a LooCI application which provides aggregated information about, and control over, all nodes in the associated WSN).

\subsection{The LooCl Binding Model}

Sections 3.1 to 3.3 introduced the supporting elements of the LooCI binding model. This section now specifically discusses the suitability of this binding model for supporting programming in WSN and other networked embedded systems. Section 3.4.1 makes the case for loosely coupled, standardized bindings. Section 3.4.2 describes the LooCI Event Ontology which supports component re-use. Section 3.4.3 gives examples of how this binding model is used. Finally, section 3.4.4 gives a preliminary sketch of how we intend to exploit this binding model to support advanced features like generic fault tolerance and distributed garbage collection.

\subsubsection{The Argument for Event-Based Bindings}

As previously described, LooCI bindings are formed by the subscription of one component to the events generated by another component. Combined with our flexible addressing scheme and connectionless network model, the result is a very loose coupling between components. LooCI bindings are asynchronous, distributed and multi-party.

- Asynchronous: event publishers do not block while producing events and subscribers are notified asynchronously when an event is received. This is an excellent fit with unreliable, resource constrained WSNs.

- Distributed: local or remote bindings are semantically identical, allowing components to be easily bound to local or remote resources, whether this is a single node, a group of nodes or a whole network.

- Multi-party: unlike traditional RPC-based approaches, which require that relationships be modeled between single nodes, LooCI bindings allow for rich interactions between nodes, groups and networks. 
The asynchronous nature of LooCI bindings is an excellent fit with resource constrained environments as program execution is not blocked during interface calls. The inherently distributed nature of our bindings allows for rich interactions between network entities to be modeled. This is in contrast to the static, local component model of NesC [6] and a significant improvement over the RPC-like bindings offered by OpenCOM and RUNES which can be used to build relationships only between single motes. LooCI components support multi-party bindings that are richer than those provided by May et al. [14], and more lightweight than the web services approach employed by Pohl et al. [3].

In a general sense, we believe that the characteristics of WSN data flows are a good fit with the features of publish-subscribe interaction models [9]. However, unlike traditional publish-subscribe systems such as Jini [25] that require specialized service brokers, LooCI is entirely decentralized, allowing any node to act as an event broker, which is critical in mobile environments where network segmentation is possible. We believe that through the provision of a per-WSN Network Manager that supports aggregate control and introspection, LooCI balances the benefits of hierarchical publish-subscribe systems with the characteristics of real world WSNs and is a clear improvement over previous interaction models.

\subsubsection{The LooCl Event Ontology}

LooCI events are defined based upon a global, hierarchical name-space represented by a spanning tree. The root of the tree is the base EVENT type and each successive layer of child nodes describes events with increased specificity. A very simple example of such an event ontology is shown in Figure 4. Events are each shown in a separate box. Parent-child relationships are shown with a single line and denote an inheritance relationship. For example, the event types TEMPERATURE and LIGHT are of type SENSOR, which itself is of type EVENT.

The structure of this tree is encoded using a unique identifier which can be used to support efficient subsumption testing - i.e. the testing of whether one event is a sub-type of another. This scheme is based upon an extension of the work reported on in [34]. The calculation of unique identifiers and the subsumption testing process are discussed in Section 3.4.2.1 and Section 3.4.2.2 respectively.

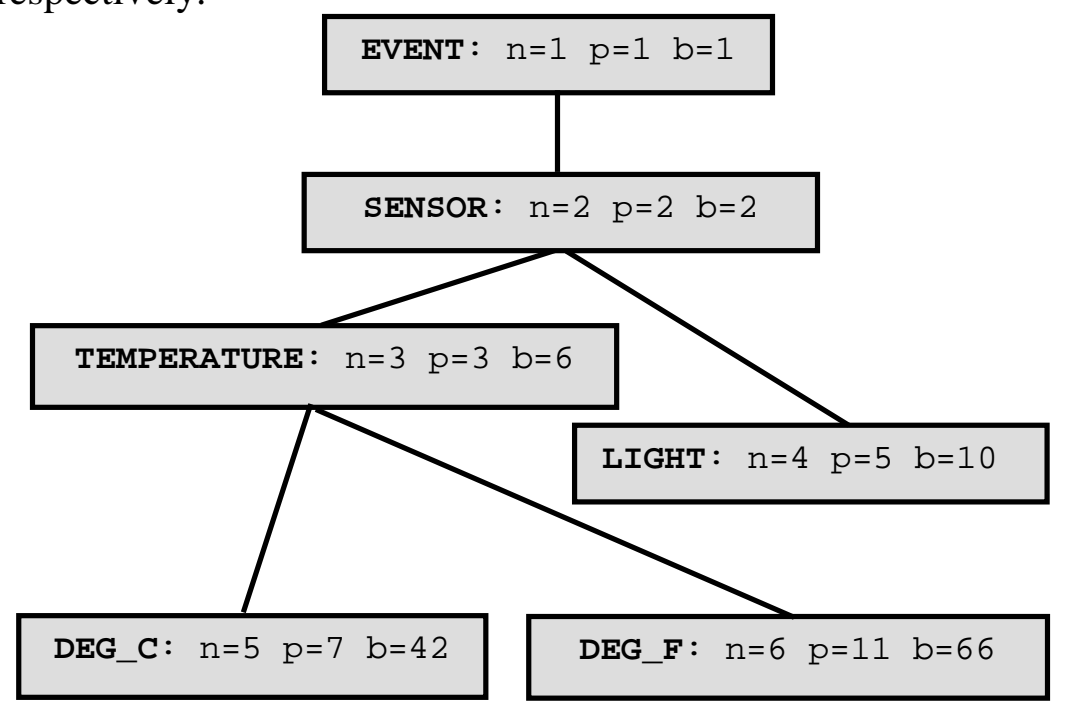

Figure 4: Example Event Ontology 


\section{Calculation of Unique Event Identifiers}

Each event in the tree is assigned two values, (n) and (p) which together can be used to efficiently compute a unique identifier (b) that is used to encode subsumption testing functionality into each event:

- n: represents the sequence number of the event as it was added to the tree. The first event is assigned the value 1 , the second event 2 and so on and so forth.

- p: is the nth prime. This is not computed dynamically, but instead is assigned based upon a well-known list of the first 10,000 primes [37].

- b: is the Globally Unique Identifier (GUID) for this event type and is calculated based upon the product of the assigned $p$-value and the $b$-value of the parent node. The root node is a special-case and is assigned the b-value 1.

This encoding scheme is very efficient, allowing for subsumption testing data to be encoded into a very compact identifier as reported in [34].

\section{Efficient Subsumption Testing}

As reported in [34], due to the properties of primes, it is easy to test whether one event $(\mathrm{X})$ is a sub-type of another, $(\mathrm{Y})$ - all that is required is to calculate $\mathrm{X}$ Mod Y. If the result of this operation is 0 , then $\mathrm{X}$ is a sub-type of $\mathrm{Y}$, otherwise $\mathrm{X}$ is not a sub-type of $\mathrm{Y}$. For example, consider the subsumption testing process to determine whether DEG_C and LIGHT are of type TEMPERATURE:

In testing whether DEG_C is a sub-type of TEMPERATURE, we perform the modulo of the b-value for DEG_C and TEMPERATURE (42 Mod 6). The result of this operation is zero and therefore DEG_C is of type TEMPERATURE.

In testing whether LIGHT is a sub-type of TEMPERATURE, we perform the modulo of the b-value of LIGHT and TEMPERATURE (10 Mod 6). The result of this operation is not zero and therefore LIGHT is not of type TEMPERATURE.

\section{Uses of Subsumption Testing}

The classification of LooCI events into an ontology has a number of advantages in terms of component re-use, binding flexibility and optimization:

In terms of re-use, a global type system makes it possible for developers to easily discover deployed 3rd party components suitable for use in their compositions. This prevents the unnecessary deployment of redundant functionality and is thus critical to conserving resources.

In terms of binding flexibility, the hierarchical event classification allows developers to easily subscribe to groups of events. For example, a temperature logging component may include temperature conversion functionality, and thus would subscribe to all events of type TEMPERATURE, rather than explicitly specifying event type DEG_C and DEG_F respectively.

This form of hierarchical classification also allows for automatic optimization of compositions at deploy-time. For example, as part of a composition, a developer may request deployment of a DEG_C component; however, a DEG_F component already exists at the desired location. In this case, the deploying agent may choose not to deploy the requested component, which would consume additional resources, but instead will wire a lightweight TEMP_CONVERSION component into the provided composition and connect this to the existing DEG_F component. While this example is trivial, we believe that in a shared 
infrastructure sensor network which supports multiple applications [20], automatic optimization of compositions may hold significant benefits.

\subsubsection{Example Bindings}

This section provides some simple examples that show how our binding model can be used to support the creation of distributed software compositions for a mobile warehouse monitoring scenario. Each example shows how the necessary bindings may be realized using LooCI API calls. Each binding is illustrated in Figure 5. In this scenario, a company, 'STORAGE_CO' uses a WSN to monitor the location of packages stored in their warehouse using RF-based localization. A sensor mote is installed in each package and the LooCI middleware runs on both WSN motes and the back-end systems of STORAGE_CO.

Example 1 - Tracking a Suspicious Package: In this example, a specific package has been tagged as 'suspicious' by a STORAGE_CO employee and its location will thus be monitored as it moves through the warehouse, until a customs officer arrives to inspect it. To support this, the SUSPECT_TRACKING component will dispatch a WIRE event to the address of the mote in the identified package. This event specifies that the LOCATION_EVENT that is produced by a LOCATION_COMPONENT running on this mote should be wired to the address of the mote hosting the VISUALISATION_COMPONENT, which will then begin to receive location telemetry from the suspect package. This is an example of how a simple one-to-one binding may be created:

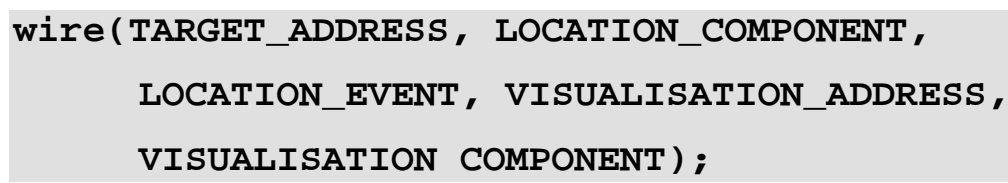

Example 2 - Emergency Data Logging: In this example, STORAGE_CO has been tasked to safely store sensitive materials, for which they must provide an unbroken location audit trail. To handle the possibility of disconnection from back-end logging systems during periods of mobility, STORAGE_CO also deploys an in-network LOGGING_COMPONENT which logs SENSOR events, storing them to flash. When the node moves out of range, the LOCATION_COMPONENT will use LooCI's introspection facilities to discover all LOGGING_COMPONENTs available on neighboring nodes, storing them to an array of addresses (logAddresses). The LOCATION_COMPONENT will then wire its LOCATION events to the addresses of each discovered LOGGING_COMPONENT:

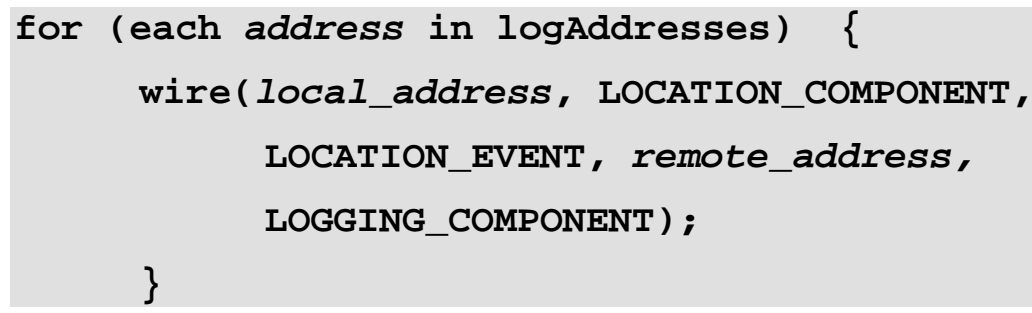

As a 'LOCATION' event is a child of the 'SENSOR' event type in the global event classification (described in section 4.3), the LOGGING_COMPONENT is implicitly subscribed to LOCATION events. This is an example of how introspection, coupled with a global type 
system can be used to support decentralized service discovery and re-use. It is also an example of a one-to-many binding. Furthermore, the LOGGING_COMPONENT provides a simple example of how hierarchical event types allow for the creation of flexible components, such as a generic logging service.

Example 3 - Filtering location data: Following deployment of a WSN and locationtracking software, STORAGE_CO discovers that their location data is subject to intermittent interference and thus inaccuracy. STORAGE_CO software engineers develop a filtering algorithm that can weed-out bogus location data. In order to install the filtering component, the Network Manager is used to discover the address of LOCATION_COMPONENTs, which is stored to an array (publishers). The Network Manager then discovers all addresses to which these LOCATION_COMPONENTs are bound:

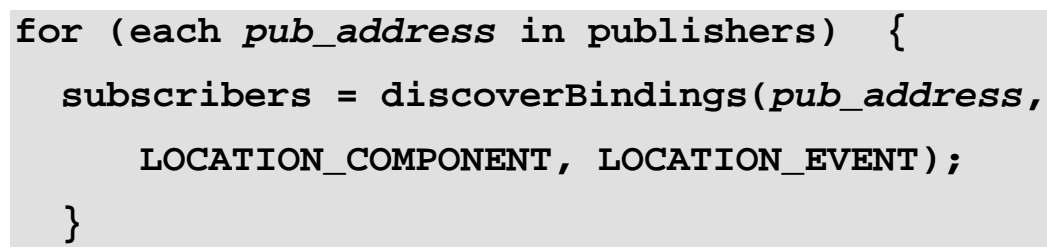

A FILTER_COMPONENT is then deployed on each mote hosting a LOCATION_COMPONENT (The FILTER_COMPONENT depends upon, and produces LOCATION events) and the LOCATION_COMPONENT is then rewired to the FILTER_COMPONENT:

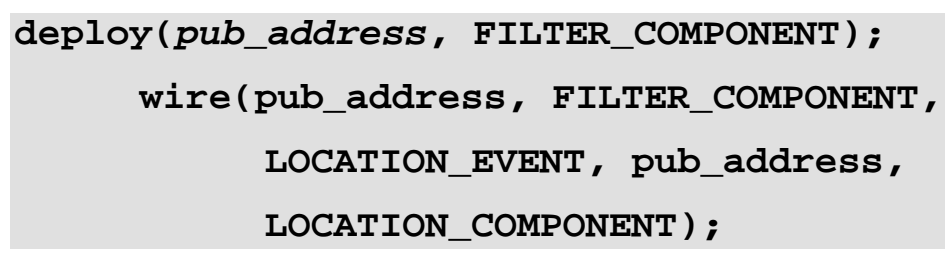

Each subscriber is then unwired from all current bindings and re-connected to the deployed FILTER_COMPONENT:

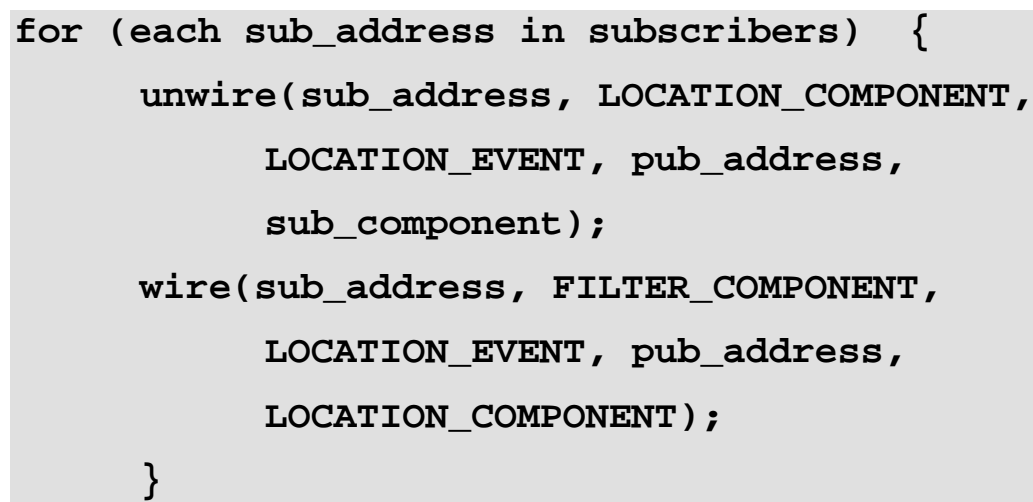

Thus all components that were previously bound to a LOCATION_COMPONENT and receiving unfiltered LOCATION events are now bound to a FILTER_COMPONENT producing 
filtered LOCATION events. This is an example of how dynamic component re-wiring can be used to modify the functionality of an existing composition. This example also shows how the loosely coupled event-bus abstraction supports easy interception of events.
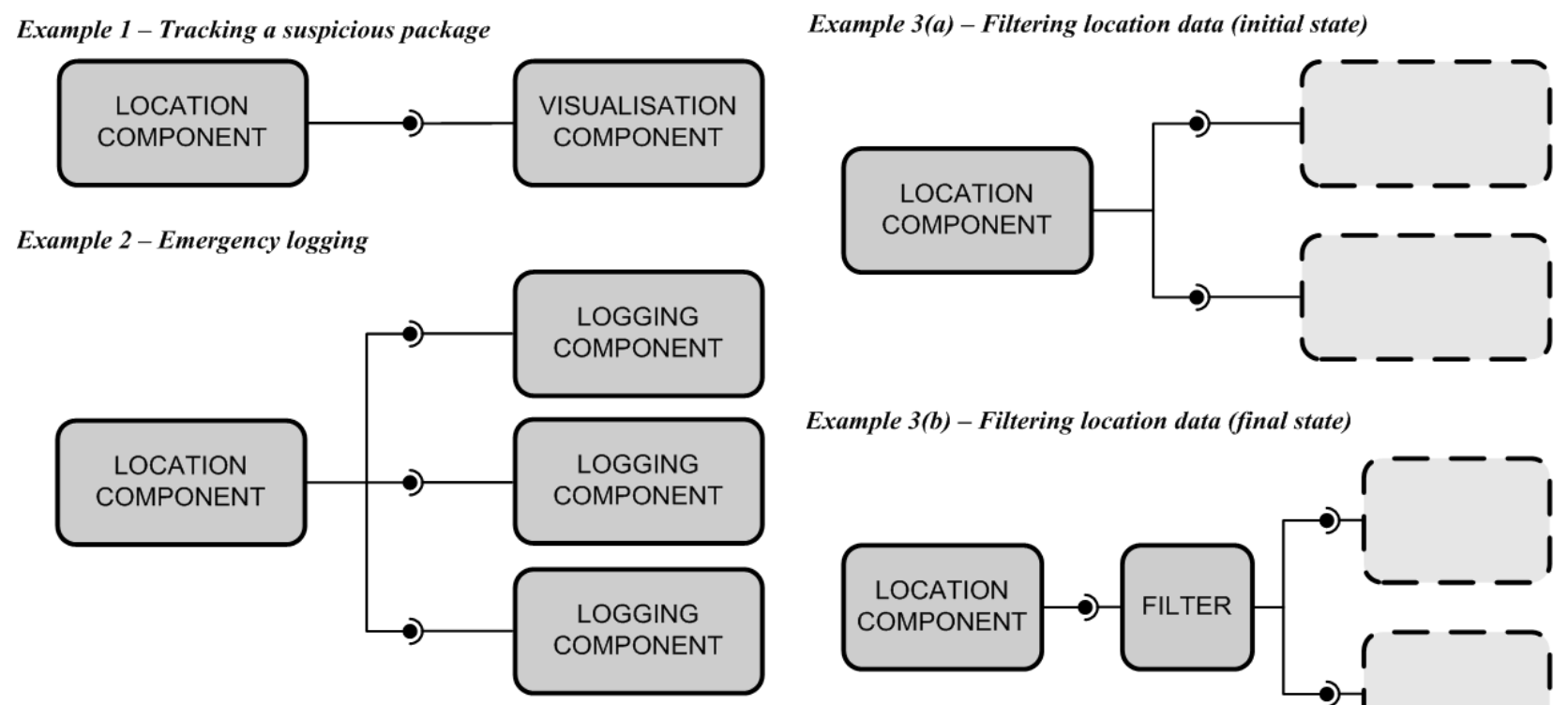

Example 3(b) - Filtering location data (final state)

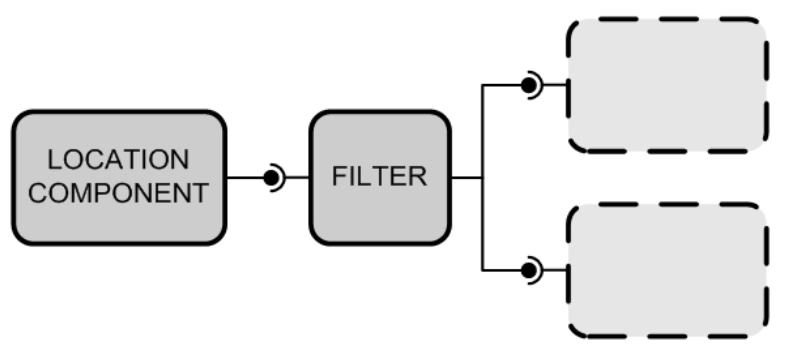

* All bindings are of type LOCATION.

Figure 5: Example LooCI bindings

\subsubsection{Supporting Advanced Features}

This section briefly discusses how the LooCI component and event model can be used to support two forms of advanced functionality: distributed garbage collection and generic fault tolerance.

Distributed garbage collection: this paper has argued that WSN are increasingly being required to support multiple applications and are being viewed as long-lived infrastructure [20]. Run-time reconfigurable component-based middleware is a good fit for this usage scenario as it allows for the modification of existing functionality along with the dynamic deployment of new functionality. However, as applications are retired and their constituent components are no longer needed, they must be removed from the network to prevent a build-up of 'orphan' components that waste resources. $\mathrm{n}$ multi-user scenarios, it may not be in the interests of a developer to invest significant time in component clean up. Alternatively, applications may crash, and go off-line in an unplanned fashion. Even where the removal of components is attempted, network failure, such as mobile nodes moving out of range, may result in incomplete clean-up of components. Fortunately, the use of a global event type system together with indirect bindings over the event bus allows for the possibility of automatic distributed garbage collection. This functionality may be realized by the Network Manager application, which, as previously stated, has responsibility for a single WSN. To accomplish this, the Network Manager periodically polls the reflective interface of each mote and component to gather deployment and wiring information. The Network Manager will also inspect the introspection interface of each remote location to which components are bound. Where no live dependent components are found, the potentially orphaned component will be flagged and, after a given time-out, will be 
garbage collected, releasing the resources it was using. If a more robust and decentralized approach is required, this functionality could also be implemented at a more fine-grained level, with multiple nodes in the WSN taking responsibility for garbage collecting unused components that are deployed on their neighbors.

Generic fault tolerance: An equally significant problem is the possibility that a mote fulfilling a binding will fail, move out of range, or become inaccessible, leaving a dependency unmet. Fortunately, the use of a global type system also allows for the implementation of generic fault-tolerance. As with distributed garbage collection, generic fault tolerance functionality may be provided by the Network Manager, which maintains a list of all of the bindings associated with all components in the WSN. The Network Manager will periodically poll the remote components participating in these bindings and, where a component appears to have failed, the manager will attempt to repair the binding. In the simplest case, this may involve rewiring to a deployed alternative component. Alternatively, the Network Manager may request the deployment of a new component that produces the required event. In this way it is possible to provide generic fault tolerance for LooCI applications. As with distributed garbage collection, where a more robust approach is required, this functionality could also be implemented in a distributed fashion, with multiple nodes in the WSN taking responsibility for repairing unmet dependencies that involve their neighbors.

Distributed garbage collection and generic fault tolerance provide specific examples of the broader advantages inherent in maintaining simple and consistent communication abstractions. These services lower the burden on the developer, while allowing her intentions to be more closely honored by the middleware. Another advanced feature of the LooCI component model that exploits the benefits of the event bus is the Policy Management Architetcure (PMA), which is discussed in section 3.5.

\subsection{Policy-Based Management for LooCl}

The Policy Management Architecture (PMA) exploits the features of the LooCI event bus to implement a simple and high level policy-based management architecture. Full details of this architecture are provided in [35] and discussed in brief below.

The specification of policies is accomplished using policy rules that follow EventCondition-Action (ECA) semantics. The semantics of these rules correspond well to the eventdriven nature of LooCI. An ECA policy consists of a description of triggering events, an optional logical expression, and a list of actions to be enforced in response. In addition, our prototype policy language allows various functions to be called inside the condition and action parts of a policy. Full details of our policy language are provided in [35], while the case-study provided in Section 6 provides concrete examples of policies.

PMA provides tool support that allows end-users to firstly select the individual components and interfaces where a policy should be applied. Secondly, after specification of the corresponding policies, the tool parses and analyzes each policy for syntactic consistency. Finally, the tool allows the end-user to choose which nodes she wants to deploy the policy to. 


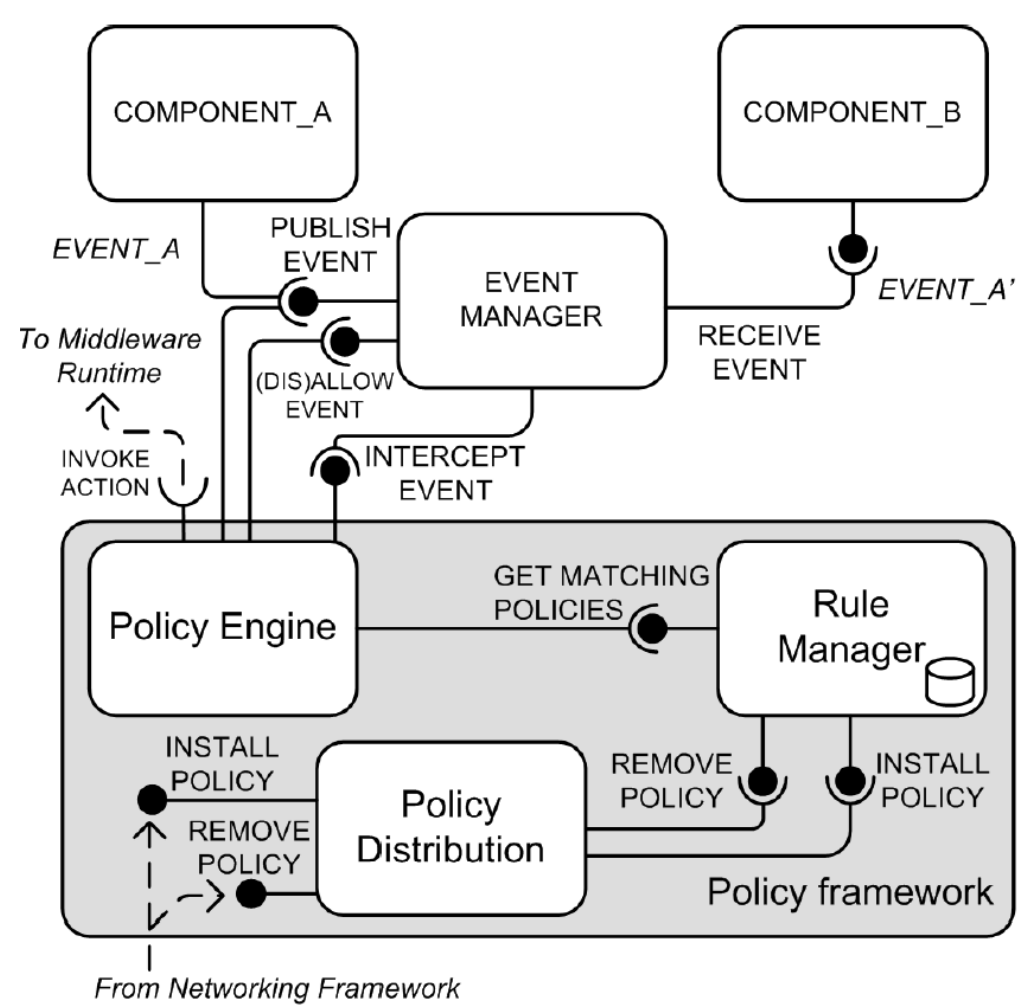

Figure 6: The Policy Based Management Architecture

\subsubsection{Policy Framework Architecture}

As illustrated in Figure 6, the policy framework may be deployed on each sensor node, the gateway, or the back-end system as needed, and consists of three key components: a Policy Engine, a Rule Manager, and a Policy Distribution component.

The Policy Engine is the main component in this framework and is responsible for intercepting events as they pass between two components and evaluating them based upon the set of policy rules on each node. In the case of a match (i.e. a triggering event and a condition evaluating to true), the engine enforces the actions defined in the action part of the matching policy. Typical examples of actions include: blocking of an event, publishing a custom event, or invoking a particular function in the middleware runtime. Potential conflicts between multiple matching policies are handled by following a priority-based ordering of policies, whereas only the actions of the policy with the highest priority are executed.

Distribution of policy files from the back-end to the sensor network is achieved using a Policy Distribution component hosted on each individual sensor node. After specification and analysis of a policy by our tool, the policy is transformed into a compact binary representation that can be efficiently disseminated to the sensor nodes. On reception of this binary policy representation, the policy distribution component passes it to the Rule Manager component.

The Rule Manager on each mote is responsible for storing and managing the set of policy rules on the node. After reception of a binary policy from the distribution component, the rule manager converts the policy into a data structure, suitable for more efficient evaluation, which is then passed to the policy engine on a per (triggering) event base. By retaining the ability to dynamically change the set of policies at runtime, the framework can be adapted to meet evolving application demands. 


\section{PERFORMANCE AND FOOTPRINT}

A prototype implementation of LooCI and PMA has been realized in Java ME for the 'RED' version of the SQUAWK JVM, which implements the Java ME CLDC 1.1 standard. This section now provides a performance and footprint analysis of LooCI and PMA. Section 6 then evaluates the functionality of LooCI in a real-world case-study.

\subsection{LooCl Memory Footprint}

It is particularly critical that middleware for embedded systems maintains a minimal memory footprint. This section compares LooCI to the GridKit middleware [10] (which uses OpenCOMJ [7]) and RUNES [4].

The complete LooCI implementation may be most directly compared to the GridKit WSN middleware which, like LooCI, offers network functionality along with support for component based software development and dynamic reconfiguration through OpenCOMJ [10]. However, it is also possible to compare a subset of the LooCI implementation (component model and reconfiguration support) to the Java implementation of the RUNES [4] component model. Table 1 compares the footprint of LooCI with GridKit and RUNES as reported in [10] and [4] respectively.

Table 1: LooCI Memory Footprint Comparison

\begin{tabular}{lccc} 
& LooCI & GridKit & RUNES \\
\cline { 2 - 4 } Core: & $20.8 \mathrm{kB}$ & $52.4 \mathrm{kB}$ & $15.5 \mathrm{kB}$ \\
Networking: & $23.4 \mathrm{kB}$ & $51.8 \mathrm{kB}$ & $\mathrm{N} / \mathrm{A}$ \\
\cline { 2 - 4 } Total: & $44.3 \mathrm{kB}$ & $104.2 \mathrm{kB}$ & $\mathrm{N} / \mathrm{A}$ \\
\hline
\end{tabular}

As Table 1 shows, the LooCI component model has a footprint of just $20.8 \mathrm{kB}$, slightly more than the RUNES component model at $15.5 \mathrm{kB}$ but significantly smaller than OpenCOMJ at $52.4 \mathrm{kB}$. Furthermore, the event bus network abstraction offered by LooCI consumes less than half of the memory of the GridKit networking framework while offering a more flexible communication abstraction. We anticipate that through further optimization, it will be possible to significantly reduce the footprint of LooCI while offering the same functionality.

LooCI components also have a minimal footprint. A null LooCI macrocomponent requires 686 bytes of disk space, while a null microcomponent consumes just 587 bytes, which is similar to a RUNES component at 544 bytes (figures are not available for an equivalent OpenCOMJ component). We anticipate that further reductions in LooCI component size may be possible through optimization of the base component class.

Table 2 compares the memory consumption of the LooCI middleware to the standard SunSPOT SDK ('RED' version). The memory consumed by a dummy component running in the master isolate (a) may be directly compared to the LooCI middleware (core and networking) running with no components registered. Thus it can be seen from the table that the LooCI runtime consumes only $7 \mathrm{~K}$ of RAM. The table also shows that running a standard SunSPOT component in a child isolate (b) increases memory consumption by $21 \mathrm{~K}$. As macrocomponents 
are instantiations of an isolate, we can expect a similar increase for each macrocomponent added. Our evaluation however shows an increase of $39 \mathrm{~K}$ for the first macrocomponent and $26 \mathrm{~K}$ for each additional macrocomponent. The disparity in overhead between SunSPOT applications and macrocomponents can be explained by the instantiation process of the inter-isolate RPC server and its proxies. Table 2 also shows that microcomponents have a much smaller memory footprint at just $2 \mathrm{~K}$ per component. Finally, it should be noted that these experiments were performed using the Solarium management tool provided as part of the SunSPOT SDK. As Solarium provides over the air monitoring of motes, it would be expected to consume a non-negligible amount of memory on each mote. However, Solarium memory consumption is constant for all experiments and thus the relative differences in memory usage shown in Table 2 remain accurate.

Table 2: LooCI Memory Usage

\begin{tabular}{cccc} 
& & \multicolumn{2}{c}{ Total RAM Used } \\
SunSPOT SDK: & (a) No Applications & \multicolumn{2}{c}{$77 \mathrm{kB}$} \\
& (b) 1 Application & \multicolumn{2}{c}{$98 \mathrm{kB}$} \\
\cline { 2 - 4 } LooCI: & No components & $84 \mathrm{kB}$ & $84 \mathrm{kB}$ \\
& 1 component & $123 \mathrm{kB}$ & $86 \mathrm{kB}$ \\
& 2 components & $150 \mathrm{~Kb}$ & $88 \mathrm{kB}$ \\
& 3 components & $176 \mathrm{kB}$ & $90 \mathrm{kB}$ \\
\hline
\end{tabular}

We evaluated LooCI on a standard SunSPOT mote (180MHz ARM9 CPU, 512KB RAM, SQUAWK VM 'RED' version) using the Solarium management application. We logged the time required to initialize the LooCI run-time, the time required to initialize a null LooCI component and the time required to send and receive an event. In each case, we performed 10 experiments. Table 3 shows the average performance characteristics observed in these experiments.

Table 3: LooCI Performance

\begin{tabular}{lcc} 
& \multicolumn{2}{c}{ Time (ms) } \\
\cline { 2 - 3 } Run-time Init: & \multicolumn{2}{c}{$498 m s$} \\
\cline { 2 - 3 } & Macro. & Micro. \\
Null-component Init: & $35 m s$ & $738 m s$ \\
Event Publication: & $14 m s$ & $4 m s$ \\
Event Reception: & $14 m s$ & $4 m s$ \\
\hline
\end{tabular}

From Table 3 it is clear that even on an embedded platform such as the Sun SPOT, the time required to initialize the LooCI runtime and LooCI components is not prohibitive, especially as such operations are likely to be infrequent. Microcomponents take significantly 
longer to initialize as they are delivered in an isolate and must first be transferred to the master isolate for execution. The performance of event dissemination for microcomponents is good; however, the performance of event publication for macrocomponents is lower due to the overhead of IIRPC calls in the SQUAWK 'RED' JVM.

\subsection{Footprint and Performance of PMA}

We also evaluated PMA on a standard SunSPOT mote (180MHz ARM9 CPU, 512KB RAM, SQUAWK VM 'RED' version) using the Solarium management application.

The resulting size of the policy framework for Sun SPOT LooCI is 26kB. Along with its compact size, the overhead of evaluating a policy and executing its actions is equal to the overhead of disseminating a LooCI event using efficient microcomponents (i.e. $4 \mathrm{~ms}$ ). The footprint and development overhead of PMA policies is analyzed in Section 5.3 and Section 5.4 respectively.

\section{CASE STUDY APPLICATION}

We evaluated LooCI in the context of a real-world river monitoring scenario in the city of São Carlos, in São Paulo State, Brazil. In this scenario, four WSN motes were deployed for two weeks at the junction of two creeks near important local infrastructure. The maximum distance between deployed nodes was 50m. The deployment topology is shown in Figure 7.

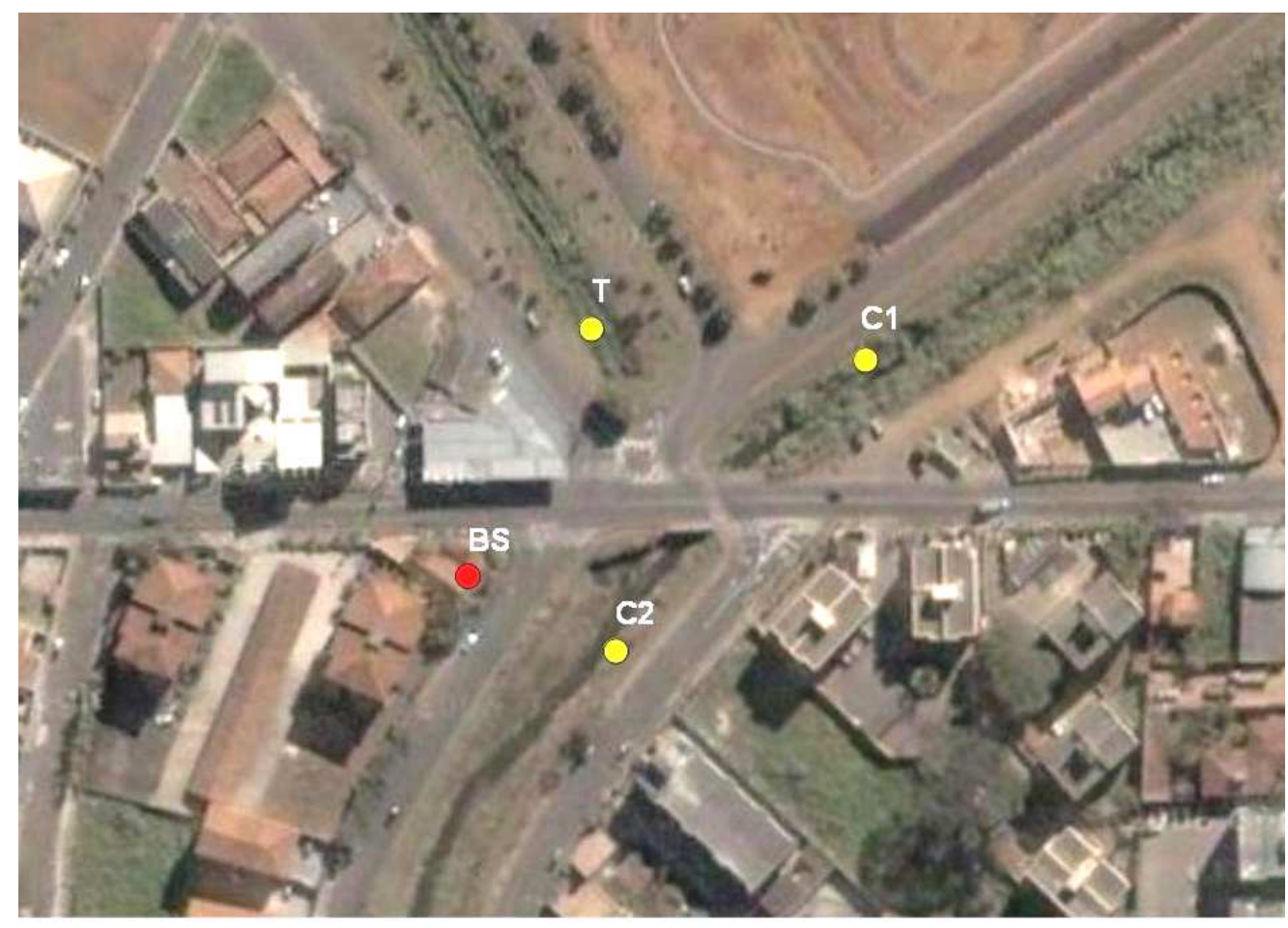

Deployment Locations: BS = Base Station, C1 = Creek 1, C2 = Creek 2, T1 = Tributary

Figure 7: Deployment Topology

The WSN mote platform employed in this system was based on the Sun SPOT platform, which was extended with a $2 \mathrm{~W}$ solar array to ensure continuous operation without battery 
replacement and additional sensors to monitor river conditions including conductivity sensors, methane sensors, hydrostatic level sensors and accelerometer-based tamper detection sensors.

The remainder of this section discusses how LooCI and PMA were used to realize this river monitoring application. Section 5.1 describes the sensor event ontology for this system. Section 5.2 reports on how LooCI components and PMA policies were used to realize application functionality. Section 5.3 analyses the footprint of the application. Finally, Section 5.4 analyses development overhead.

\subsection{Event Ontology}

The portion of the event ontology that relates to sensor events is shown in Figure 8 along with the unique identifiers that are assigned to each event (b-values). For space reasons, the complete ontology is not reproduced here. This event taxonomy is exploited in the application composition described in Section 5.2 to allow for the creation of a generic Environmental Hazard Alert component. This component is used to display readings from diverse sensors types using a single receptacle.

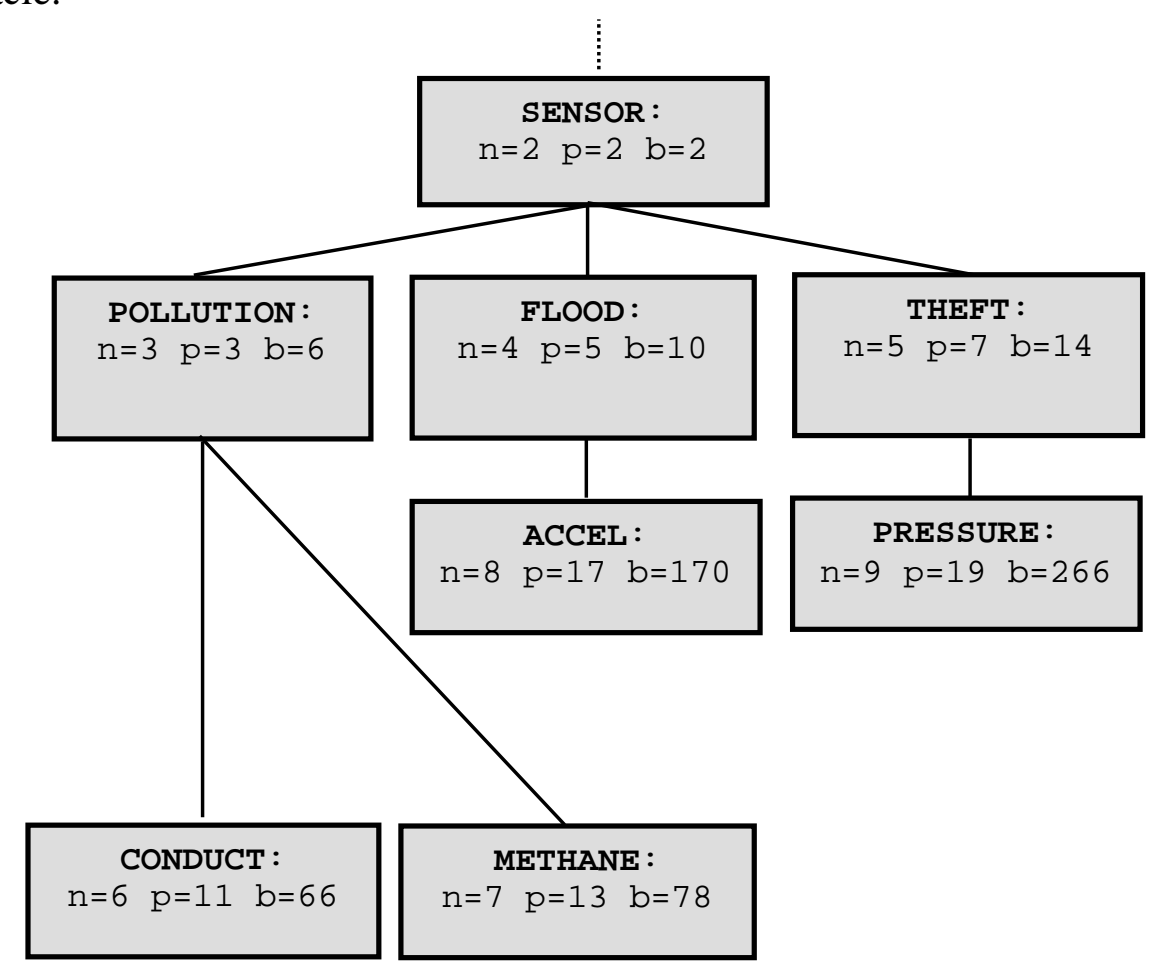

Figure 8: Event Ontology

\subsection{Application Composition}

The river monitoring application composition consists of five components and three policies, the functionality of which is outlined below:

\section{COMPONENTS:}

- ConductivitySensor: the ConductivitySensor component exposes readings from the water conductivity sensor via a single interface of type CONDUCT $(n=6)$. 
- MethaneSensor: the MethaneSensor component exposes readings from the $\mathrm{CH}_{4}$ sensor via a single interface of type METHANE ( $\mathrm{n}=7$ ).

- AccelerometerSensor: the AccelerometerSensor component exposes readings from the Sun SPOT accelerometer via a single interface of type ACCEL ( $\mathrm{n}=8)$.

- PressureSensor: the PressureSensor component exposes readings from the vented gauge hydrostatic level sensor via a single interface of type PRESSURE $(n=9)$.

- EnvironmentalHazardAlert: the EnvironmentalHazardAlert component offers a single receptacle of type SENSOR $(n=2)$ which displays environmental sensor readings in a graphical user interface. Due to the properties of the event ontology this component will display readings from all deployed sensing components (IDs 6 through 9 in Figure 8).

Components are wired as shown in Figure 8. Lines ending in balls denote interfaces, while lines ending in cups denote receptacles.

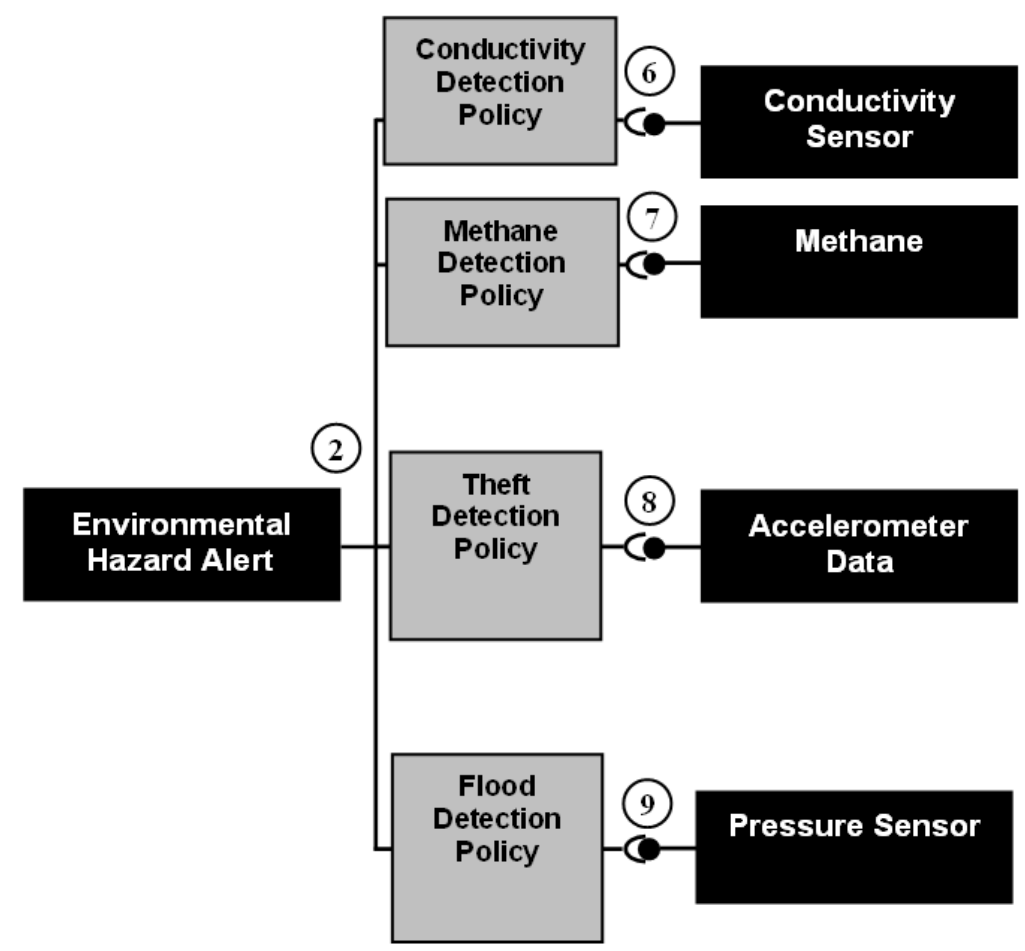

Figure 9: Application Composition

\section{POLICIES:}

- ConductivityDetectionPolicy: this policy effectively acts as a filter, allowing CONDUCTIVITY (6) events to pass to the EnviornmentalHazardAlertComponent only where the reading contained in the EVENT exceeds a specified threshold (and thus indicates that a conductive pollutant has entered the river). This threshold value is configured at the level of individual motes, based upon previously observed readings.

- MethaneDetectionPolicy: this policy effectively acts as a filter, allowing METHANE (7) events to pass to the EnviornmentalHazardAlertComponent only where the reading contained in the EVENT exceeds a specified threshold (and thus indicates that an organic pollution event has occurred). This threshold value is also configured at the level of individual motes, based upon previous readings. 
- TheftDetectionPolicy: this policy filters ACCEL (8) readings, only allowing them to pass to the EnviornmentalHazardAlertComponent where they exceed a threshold that indicates theft or vandalism. This threshold is initially configured globally for every mote, but may be tailored at run-time to account for natural movement of the mote (for example due to trees swaying in the wind).

- FloodDetectionPolicy: this policy filters PRESSURE (8) readings, only allowing them to pass to the EnviornmentalHazardAlertComponent where they exceed a threshold that indicates flooding. This threshold value is configured at the level of individual motes, based upon the height of the river bank at that deployment location, however, this value may be tweaked to account for bank erosion or reinforcement.

In terms of composition functionality, all sensing components $(n=6, n=7, n=8$ and $n=9)$ are wired to the Environmental Hazard Alert component $(n=2)$. Without the intervention by the policy engine, the sensing components will transmit all sensor readings to the alert system as parameterized; however, the policies described previously act as filters, only allowing SENSOR events to reach the hazard alert system where they reach some pre-defined threshold.

In general terms, it should be apparent from Figure 8 that much of the functionality in our composition is re-usable. For example, the sensor components could be used in any other application that required environmental data of this type. Furthermore, the functionality of these components can easily be discovered at run-time using the LooCI event ontology and thus included in other application compositions.

\subsection{APPLICATION FOOTPRINT}

For resource constrained embedded systems, maintaining a minimal application footprint is critical for two primary reasons: Firstly, a small application footprint leaves more space available for data logging. Secondly, in the case of reconfigurable systems, where component updates may be transmitted over the air, a smaller component footprint reduces radio activity and thus conserves battery life.

The foot-print of all components in our application composition are shown in Table 4. As can be seen from the table, all components have a footprint of less than $2 \mathrm{kB}$, with the exception of The EnvironmentalHazardAlert component that runs on the resource-rich back-end (and therefore, its larger size has no significant impact on system performance).

Table 4: Component Footprint

\begin{tabular}{rc}
\cline { 2 - 2 } ConductivitySensor: & Size \\
MethaneSensor: & $1.8 \mathrm{kB}$ \\
PressureSensor: & $1.7 \mathrm{kB}$ \\
AccelerometerSensor: & $1.7 \mathrm{kB}$ \\
Total: & $1.9 \mathrm{kB}$ \\
\cline { 2 - 2 } & $13.2 \mathrm{kB}$ \\
\hline
\end{tabular}


Table 5: Policy Footprint

\begin{tabular}{rc}
\cline { 2 - 2 } ConductivityDetectionPolicy: & Size \\
MethaneDetectionPolicy: & 94 bytes \\
TheftDetectionPolicy: & 94 bytes \\
FloodDetectionPolicy: & 95 bytes \\
Total: & 94 bytes \\
\cline { 2 - 2 } & 377 bytes
\end{tabular}

The foot-print of all policies in our application composition are shown in Table 5. As can be seen from the table, policies are much more compact than components, with all policies consuming between 94 bytes and 95 bytes - an order of magnitude less than components. This is particularly advantageous as policies are used to represent dynamic concerns such as alert thresholds and are therefore more likely to be updated at run-time over-the-air.

This section provides a quantitative assessment of development overhead. Table 6 and Table 7 respectively provide a Lines of Code (LoC) analysis for all components and policies in our application.

As can be seen from Table 6, LooCI components are relatively compact and impose limited overhead on developers in terms of LooCI-specific code. The identical size of the first three components listed in the table may be attributed to their simplicity and similarity (in all cases, these components read a simple value from the Sun SPOT Analogue to Digital Converter (ADC) and transmit it over the event bus).

Table 6: Lines of Code: Components

\begin{tabular}{|c|c|c|}
\hline ConductivitySensor: & $\begin{array}{c}\text { Functional } \\
\text { Code } \\
48\end{array}$ & $\begin{array}{c}\text { LooCI } \\
\text { Code } \\
11\end{array}$ \\
\hline MethaneSensor: & 48 & 11 \\
\hline PressureSensor: & 48 & 11 \\
\hline AccelerometerSensor: & 42 & 11 \\
\hline EnvironmentalHazardAlert*: & 41 & 23 \\
\hline Total: & 227 & 67 \\
\hline
\end{tabular}

The high-level nature of PMA policies allows rich and compact expression of both functional and non-functional objectives. Complemented with a demonstrated lightweight representation and enforcement mechanism, policies are well suited to accommodate the dynamic concerns in WSNs. 
Table 7: Lines of Code: Policies

\begin{tabular}{rc}
\cline { 2 - 2 } ConductivityDetectionPolicy: & Size \\
MethaneDetectionPolicy & 10 \\
TheftDetectionPolicy: & 10 \\
FloodDetectionPolicy: & 10 \\
Total: & 10 \\
\cline { 2 - 2 } & 40
\end{tabular}

\section{FUTURE WORK}

Our future work will focus upon three key areas: (i) extension of the LooCI network framework, (ii) porting LooCI to the Contiki [18] and OSGi [5] platforms and (iii) implementation of distributed garbage collection and generic fault tolerance strategies.

The current implementation of the LooCI networking framework provides support for unicast, broadcast and neighbourcast as discussed in section 3.2. However, we believe that support for more flexible group messaging and multicast are essential to support efficient communication in challenging network environments. We also intend to develop an implementation of LooCI for the Contiki platform which will allow us to support more embedded mote platforms [21] and the OSGi platform [18], which will allow us to support standard PCs and powerful embedded devices. Another key objective in the short-term is to realize and evaluate the distributed garbage collection and generic fault tolerance functionality introduced in section 3.4.3.

\section{CONCLUSIONS}

This paper described LooCI, a novel component and binding model for WSNs and its associated policy-based management framework. Key features of LooCI are: support for runtime reconfiguration, introspection and low development overhead. We also described PMA, a high-level policy-based management architecture for LooCI. We argue that LooCI's loosely coupled, globally typed event bus is a good fit for implementing component bindings in WSN environments that are inherently asynchronous and unreliable.

The good performance characteristics and minimal memory footprint of LooCI have been demonstrated in Section 4, while a real-world case study has been used to demonstrate the appropriateness and efficiency of our development model in Section 5.

\section{ACKNOWLEDGEMENTS}

Research for this paper was partially funded by IMEC and the Instituut voor de Aanmoeding van Innovatie door Wetenschap en Technologie in Vlaanderen (IWT). This research is conducted in the context of the IWT-STADIUM project No. 80037 [28]. Wouter Horré is a PhD fellow of the Research Foundation - Flanders (FWO).

Dr. Danny Hughes and Dr. Jo Ueyama would like to thank FAPESP for funding the casestudy elements of this research project (Process ID 08/05346-4 and 09/01881-5). 


\section{REFERENCES}

[1] Mainwaring A., Polastre J., Szewczyk R., Anderson J., (2002), Wireless Sensor Networks for Habitat Monitoring, in Proc. of $1^{\text {st }}$ ACM International Workshop on Wireless Sensor Networks and Applications, Atlanta, Georgia, USA, pp 88 - 97.

[2] Hughes D., Greenwood P., Coulson G., Blair G., Pappenberger F., Smith P., Beven K., (2007), An Experiment with Reflective Middleware to Support Grid-based Flood Monitoring, in Wiley Inter-Science Journal on Concurrency and Computation: Practice and Experience, vol. 20, no 11, pp 1303-1316.

[3] Pohl A., Krumm H., Holland F., Stewing F. J., Lueck I., (2008), Service-Orientation and Flexible Service Binding in Distributed Automation and Control Systems, in Proc. of the $22^{\text {nd }}$ International Conference on Advanced Information Networking and Applications - Workshops (IANA), Okinawa, Japan, pp. 1393 - 1398.

[4] Costa P., Coulson G., Gold R., Lad M., Mascolo C., Mottola L., Picco G.P., Sivaharan T., Weerasinghe N., Zachariadis S., (2007), The RUNES Middleware for Networked Embedded Systems and its Application in a Disaster Management Scenario, in Proc. of the 5th Annual IEEE International Conference on Pervasive Computing and Comunications (PerCom'07), White Plains, New York, pp. $69-78$.

[5] Rellermeyer J., Alonso G., (2007), Concierge: A Service Platform for Resource-Constrained Devices, in ACM SIGOPS Operating Systems Review, Vol. 41, No. 3, pp. 245 - 258

[6] Gay D., Levis P., Von Behren R., Welsh M., Brewer E., Culler D., (2003), The NesC Language: A Holistic Approach to Networked Embedded Systems, in Proc. of the conference on Programming Language Design and Implementation, ACM SIGPLAN, San Diego, California, USA, pp. $1-11$.

[7] Coulson G., Blair G., Grace P., Taiani F., Joolia A., Lee K., Ueyama J. and Sivaharan T., (2008), A Generic Component Model for Building Systems Software, in ACM Transactions on Computer Systems, Vol. 26, No. 1.

[8] Bell M., (2008), Introduction to Service-Oriented Modeling, Service-Oriented Modeling: Service Analysis, Design, and Architecture, Wiley \& Sons, pp. 1 - 27.

[9] Eugster P. T., Felber P. A., Guerraoui R., Kermarrec A. M., (2003), The Many Faces of Publish Subscribe, in ACM Computing Surveys (CSUR), Vol. 35, No. 2, pp. 114-131.

[10] Grace P., Hughes D., Porter B., Blair G., Coulson G., Taiani F., (2008), Experiences with Open Overlays: A Middleware Approach to Network Heterogeneity, in Proc. of the European Conference on Computer Systems (EuroSys'08), Glasgow, Scotland, UK, pp. 123-136.

[11] Sun Microsystems, Small Programmable Object Technology, Inspiring Java developers to create a whole new breed of devices and technologies - and accelerating the growth of the 'Internet of Things': http://www.sunspotworld.com/vision.html

[12] Hill J., Szewczyk R., Woo A., Hollar S., Culler D., Pister K., (2000), System Architecture Directions for Networked Sensors, in ACM SIGPLAN, Vol. 35, No. 11, pp. 93-104.

[13] Buonadonna P. Hill J., Culler D., (2001), Active Message Communication for Tiny Networked Sensors, in Proc. of the $20^{\text {th }}$ annual Joint Conference of the IEEE Computer and Communications Societies (InfoCom'01), Anchorage, Alaska, USA, $1-11$.

[14] May T. D., Dunning S. H., Hallstrom J. O., (2005), An RPC Design for Wireless Sensor Networks, in Proc. of the IEEE International Mobile Adhoc and Sensor Systems Conference, (MASS'05), Washington, DC, USA, pp. 138-146.

[15] Smith P., Hughes D., Beven K., Cross P., Tych W., Coulson G., Blair G., (2009), Towards the Provision of Site Specific Flood Warnings using Wireless Sensor Networks, in Wiley Inter-Science journal on Meteorological Applications, Vol. 16, No.1, pp. $57-64$.

[16] MoteIV, T-MOTE Sky Ultra-low Power Wireless Module Data Sheet: http://www.cs.uvm.edu/ crobinso/mote/tmote-sky-datasheet-102.pdf

[17]Crossbow, MICA-Z Wireless Measurement System, Data Sheet: http://www.xbow.com/Products/Product_pdf_files/Wireless_pdf/MICAz_Datasheet.pdf

[18] Dunkels A., Grönvall B., Voigt T., (2004), Contiki - a Lightweight and Flexible Operating System for Tiny Networked Sensors, in Proc. of 29 th IEEE International Conference on Local Computer Networks (LCN'04), Tampa, FL, USA, pp. $455-462$.

[19] Parlavantzas N., Coulson G., Blair G., An Extensible Binding Framework for Component-Based Middleware, in Proc. of the 7th IEEE International Enterprise Distributed Object Computing Conference (EDOC'03), Brisbane, Australia, September 2003, pp 252 - 263.

[20] Huygens C., Joosen W., (2009), Federated and Shared Use of Sensor Networks through Security Middleware, in Proc. of the 6th International Conference on Information Technology: New Generations (ITNG'09), Las Vegas, Nevada, USA, pp. 1005-1011.

[21] Sentilla, Perk Platform Frequently Asked Questions: http://www.sentilla.com/perk_faq.html

[22] Sun Microsystems, Java ME - the Most Ubiquitous Application Platform for Mobile Devices: http://java.sun.com/javame/index.jsp

[23] IONA et al., Service Component Architecture: Building Systems using a Service Oriented Architecture: www.iona.com/devcenter/sca/SCA_White_Paper1_09.pdf

[24] Grace P., Coulson G., Blair G., Porter B., Hughes D., (2006), Dynamic Reconfiguration in Sensor Middleware, in Proc. of the $1^{\text {st }}$ International Workshop on Middleware for Sensor Networks (MidSens'06), Melbourne, Australia, pp. $1-6$. 
[25] Simon D., Cifuentes C., Cleal D., Daniels J., White D., (2006), Java on the Bare Metal of Wireless Sensor Devices: the Squawk Java Virtual Machine, in Proc. of the $2^{\text {nd }}$ International Conference on Virtual Execution Environments, Ottawa, Canada, pp $78-88$.

[26] Lee s., Lee Y., Lee H., (2006), Jini-Based Ubiquitous Computing Middleware Supporting Event and Context Management Services in Lecture Notes in Computer Science, Vol. 4159 No. 6, pp. 786-795

[27] Costa P., Mottola L., Murphy A.L., Picco G.P, (2007), Programming Wireless Sensor Networks With the TeenyLime Middleware, in Proc. of the ACM/IFIP/USENIX International Conference on Middleware, (Middleware'07), Newport Beach, California, pp. 429-449.

[28]IWT Stadium project 80037, software technology for adaptable distributed middleware: http://distrinet.cs.kuleuven.be/projects/stadium/

[29] Preuveneers D., Berbers Y., (2008), Encoding Semantic Awareness in Resource-Constrained Devices, in IEEE Intelligent Systems, Vol. 23, No. 2, pp. 26-33.

[30] Dowling J., Cahill V., (2004), Self-Managed Decentralised Systems using K-components and Collaborative Reinforcement Learning, in proc. of $1^{\text {st }}$ ACM SIGSOFT workshop on Self-managed Systems (WOSS'04), pp. 39-43.

[31] Rellermeyer J., Alonso G., Roscoe T., (2007), R-OSGi: Distributed applications through software modularization. In proc. of $8^{\text {th }}$ International Middleware Conference (Middleware'07), pp. 1-10.

[32] Pohl A., Krumm H., Holland F., Stewing F. J., Lueck I., (2008), Service-Orientation and Flexible Service Binding in Distributed Automation and Control Systems, in proc. of the $22^{\text {nd }}$ International Conference on Advanced Information Networking and Applications (IANA), Okinawa, Japan, March 2008, pp. 1393-1398.

[33] Marron P..J., Lachenmann A., Minder A., Hahner J., (2005), Sauter R., Rothermel K, TinyCubus: A flexible and adaptive framework for sensor networks, in proc. of the $2^{\text {nd }}$ European Workshop on Wireless Sensor Networks (EWSN), pp. 278-289.

[34] Preuveneers D., Berbers Y., (2008), Encoding Semantic Awareness in Resource-Constrained Devices, in IEEE Intelligent Systems, vol. 23, no. 2, pp 26-33.

[35] Matthys N., Huygens C., Hughes D., Michiels S., Joosen W., (2009), Flexible Integration of Data Qualities in Wireless Sensor Networks, in the proceedings of the 4th International Workshop on Middleware Tools, Services and Run-Time Support for Sensor Networks (MidSens'09), Urbana Champaign, Illinois, USA..

[36] Smith P., Hughes D., Beven K., Cross P., Tych W., Coulson G., Blair G., (2009), Towards the Provision of Site Specific Flood Warnings using Wireless Sensor Networks, in the Inter-Science journal on Meteorological Applications, Vol. 16, No.1, pp. 57-64.

[37] The University of Tenessee at Martin, The First 10,000 Primes, available online at: http://primes.utm.edu/lists/small/10000.txt. 